\title{
Microstructural Studies of Carbon Black Filler in Standard Malaysia Rubber Grade L (SMRL)
}

\author{
Mohamad Deraman, Wan Manshol Wan Zin, J. W. White, ${ }^{*}$ \\ S. J. Henderson, ${ }^{*}$ Salleh Nordin, ${ }^{* *}$ J. B. GomeZ,** \\ Samsidar HAMZAH, ${ }^{* *}$ and W. WAGNER ${ }^{* * *}$ \\ Nuclear Energy Unit, Kompleks PUSPATI, \\ 43000 Kajang, Malaysia \\ * Research School of Chemistry, Australian National University, \\ Canberra, A.C.T., 2601, Australia \\ ** Rubber Research Institute of Malaysia, 47000 Sungai Buloh, \\ Selangor, Malaysia \\ ***Hahn-Meitner Institut fur Kernforschung, Berlin, Glienicker \\ Str. 100, 1000 Berlin, Germany
}

(Received October 6, 1989)

\begin{abstract}
Microstructure of carbon black aggregates both before and after incorporation in natural rubber have been investigated using a small angle X-ray scattering (SAXS) and an electron microscopy techniques. Both techniques suggest that there is no change in the average diameter and shape of carbon black aggregates after their incorporation in rubber. Aggregates appear to have a fractal structure.
\end{abstract}

KEY WORDS Natural Rubber / Carbon Black Filler / Vulcanization / Gamma Irradiation / Small Angle X-Ray Scattering / Transmission Electron Microscopy / Microstructure of Filler / Fractal Structure /

The characterization of filler particles used for reinforcement purpose, in rubber has been a subject of interest amongst a large number of researchers, in particular, polymer scientists. The amount of filler added into rubber and the subsequent treatment used will depend on the desired properties of the end product. An analytical technique, namely transmission electron microscopy (TEM), has been widely used as a standard tool for studying the characteristics of filler particles in rubber. However in recent years, another technique, known as a small angle X-ray scattering (SAXS) has also been used for such an investigation.

Carbon black of different types have been succesfully used as reinforcement filler in rubber products. ${ }^{1}$ Its characteristics before and after incorporation in natural rubber have previously been studied in terms of the average particle size, size distribution, volume fraction, surface area and morphology for varying amounts of particles used and different types of treatment procedures. For example, Medalia et al. ${ }^{2}$ have reported in detail the characteristics of aggregates of carbon black particles using TEM. Recently, Young et al. ${ }^{3}$ have studied the comparison between the characteristics of carbon black and silica fillers of different concentrations in the Standard Malaysia Rubber Grade L (SMRL) samples using both SAXS and TEM instruments as the analytical tools. They claimed that their group was the first to employ SAXS for such an investigation, and found that (i) scattering data obtained from a slit collimation have a slope of -3 , which is equivalent to -4 for a pin-hole collimation (ii) a significant change in the 
microstructure of carbon black particles occurs after incorporation into rubber, i.e., the average particles diameter increased by nearly a factor of two independent of the filler concentration used, 10, 20,30, and $40 \mathrm{phr}$ (phr, parts by weight of filler per hundred parts of rubber).

As the significant change in the microstructure of carbon black filler, which occur in the SMRL sample are independent of the filler concentration, ${ }^{3}$ we therefore decided to limit our microstructural investigation for SMRL samples containing only one value of filler concentration that is $20 \mathrm{phr}$. TEM and SAXS measurements were carried out on samples prepared following the treatment procedure employed by Young et al. ${ }^{3}$ In addition to this, irradiation treatment which employed gamma ray was used for curing samples. Furthermore, we also carried out experiments before and after the samples were treated for curing. Measurement on SMRL samples without carbon black was only made by SAXS since the scatterers in the samples, which may consist of voids, are not visible by TEM.

\section{THEORETICAL FORMALISM OF SAXS}

The theoretical treatmment of SAXS in now well established. ${ }^{4}$ It is known that most of the information about the structure of a sample, within a size range from $1 \mathrm{~nm}$ to $100 \mathrm{~nm}$, can be found from the scattering function over scattering angles which satisfy the condition.

$$
0.1 \leqslant Q D \leqslant 10
$$

where $D$ is a dimension characterising the scattering object in the sample, and $Q$ is the momentum transfer which varies with scattering angle $\theta$ according to equation

$$
Q=\frac{4 \pi}{\lambda} \sin (\theta / 2)
$$

For convenience, the scattered intensity is usually expressed as a function of momentum transfer $Q$. Guinier's and Porod's laws have frequently been used in the interpretation of the scattered intensity in low and high $Q$-regions respectively. For a pinhole collimation, Guinier's and Porod's, ${ }^{6}$ laws are respectively given by

$$
I(Q)=N(\Delta \rho)^{2} V^{2} \exp \left(-Q^{2} R_{\mathrm{g}}{ }^{2} / 3\right)
$$

and

$$
I(Q)=2(\Delta \rho)^{2} S Q^{\mathrm{n}}
$$

where $N$ is the number of scatterers per unit volume, $\Delta \rho$ is the electron density difference between the scatterers and the matrix, $V$ is the scatterers volume, $R_{\mathrm{g}}$ is the radius of gyration of the scattering object, $S$ is the total surface area of scatterers in the sample, and $n$ is a characteristic exponent which determines the shape of the scattering object; spheres $(n=-4)$, ellipsoids $(n=-2$ to -4$)$, plates $(n=-2)$, needles $(n=-1)$, and fractals ${ }^{7}(n=-1$ to -3$)$. Another method which can also be used to evaluate scattering data is called the dynamical scaling procedure proposed by Fratzl et al. ${ }^{8}$ This procedure suggests that the principle morphologies of the scatterers in different samples are similar if their scattering curves in double-log presentation have an equivalent shape. Similarity in shape can be checked by shifting the frames along the direction parallel to absissa and ordinate. Application of this procedure, for example, has been published by Deraman et al. ${ }^{9}$ All of the three methods described above will be used in the interpretation of our SAXS data.

\section{EXPERIMENTAL METHODS}

Table I lists all the samples which have been investigated. The carbon black used were of HAF type (Mechem N330, ASTM No. 330). Standard Malaysia Rubber grade L (SMRL), a crosslinking agent dicumyl peroxide and filler particles, namely carbon black were mechanically mixed by a two-roll mill machine. These mixed samples were moulded to a size of $10 \mathrm{~cm} \times 10 \mathrm{~cm} \times 2 \mathrm{~mm}$, and heated at $100^{\circ} \mathrm{C}$ for 
Table I.

\begin{tabular}{ccc}
\hline & Aggregate & \\
Sample/treatment & number per & Porod slope \\
& sq. $/ \mu \mathrm{m}$ & (SAXS) \\
& (TEM) &
\end{tabular}

\begin{tabular}{|c|c|c|}
\hline A. Carbon black & - & 3.5 \\
\hline \multicolumn{3}{|c|}{ B. SMRL +1 phr dicumyl peroxide } \\
\hline Cured & - & 2.5 \\
\hline \multicolumn{3}{|c|}{$\begin{array}{l}\text { C. SMRL }+1 \text { phr Dicumyl peroxide }+20 \mathrm{phr} \\
\text { carbon black }\end{array}$} \\
\hline (i) Uncured & - & -3.4 \\
\hline (ii) Cured & 45 & -3.8 \\
\hline (iii) Irradiated & 38 & -3.6 \\
\hline \multicolumn{3}{|c|}{ D. SMRL +20 hpr Carbon black } \\
\hline (i) Uncured & 38 & -3.4 \\
\hline (ii) Irradiated & 41 & -3.4 \\
\hline
\end{tabular}

$10 \mathrm{~min}$ in order to allow the sample to achieve a good isotropic state. Two samples were treated only up to this stage. These are called uncured samples and are labelled $\mathrm{C}(\mathrm{i})$ and $\mathrm{D}(\mathrm{i})$. For samples $\mathrm{B}$ and $\mathrm{C}(\mathrm{ii})$, they were followed by a heating at $160^{\circ} \mathrm{C}$ for $50 \mathrm{~min}$. This was done in order to achieve a vulcanization state and we call these samples as cured samples. Vulcanization for samples C(iii) were achieved by irradiating them with $3 \mathrm{Mrad}$ gamma irradiation using Co-60 at the Nuclear Energy Unit, Malaysia.

The SAXS measurements were made on each sample listed in Table I using the one metre Huxely Holmes Camera in the Research School of Chemistry, A.N.U., Australia. This instrument consists of $\mathrm{GX} 13$ rotating anode X-ray generator, bent quartz crystal monochromator, a linear position sensitive proportional counter, and an on line microcomputer for data acquisition and analysis. The dimensions of rubber samples were about $0.5 \mathrm{~cm} \times 0.5 \mathrm{~cm} \times$ $0.5 \mathrm{~mm}$, obtained by cutting bigger samples explained above. For carbon black samples, the powder were filled in between two $10^{-3}$ inches thick MYLAR sheets transparent to $\mathrm{X}$-rays, giving a sample thickness of about $1 \mathrm{~mm}$. SAXS data were collected, after the corrections for transmission, background counts and sample thickness, in the form of
$\mathrm{X}$-ray intensity $\left(\mathrm{Xs}^{-1}\right)$ versus scattering angle corresponding to a $Q$ range from $0.009 \AA^{-1}$ to $0.4 \AA^{-1}$.

TEM of RRIM were used for investigation on sample B, C(i), (ii), (iii) and D(i) and (ii). TEM samples were prepared by taking $1 \mathrm{~mm}^{3}$ of material from each of the original $10 \mathrm{~cm} \times 10 \mathrm{~cm} \times 2 \mathrm{~mm}$ samples. These cubes were then fixed in $1 \%$ osmium tetraoxide for $24 \mathrm{~h}$. The samples were first washed with distilled water before they were brominated for $3 \mathrm{~h}$. This was followed by washing, post-fixing in osmium tetraoxide for another $24 \mathrm{~h}$ and washing again. Dehydration was carried out through a graded alcohol series, after which they were put in $50: 50$ alcohol $(100 \%)$ : styrene methacrylate for $15 \mathrm{~min}$. The samples were then embedded straight away (i.e., in $100 \%$ styrene methacrylate) and polymerised in the oven $\left(60^{\circ} \mathrm{C}\right)$ for $48 \mathrm{~h}$. This was found most suitable as the normal procedure caused excessive swelling of the samples.

Thin sections obtained by using a glassknived ultramicrotome were examined unstained with a Philips EM 300. The number of aggregates/sq. $\mu \mathrm{m}$ was determined by two operators from randomised areas on the micrographs. The means were calculated and listed in Table I.

Independent TEM measurements using TEM of University of Sydney, Australia, were conducted on samples A and C(iii). Sample preparation for these measurements differ from that made for TEM of RRIM, and were made as follows: The samples, $2 \mathrm{~mm} \times 10 \mathrm{~mm} \times 10$ $\mathrm{mm}$, taken from the original sample, were cut and dropped into liquid nitrogen and left to equilibrate for one hour. Sections were cut at $-70^{\circ} \mathrm{C}$ on glass knives in a Reichert FC4 attachment on the ultramicrotome. These were first scooped onto 200 mesh glow discharge treated grids which had been coated with parlodion and carbon, and then stamped with a teflon rod to flatten the sections. The grids were then withdrawn and allowed to come to room temperature in a dry atmosphere. 


\section{RESULTS AND DISCUSSION}

The scattering from the aggregate of carbon black particles in samples $\mathrm{C}$ and $\mathrm{D}$ were obtained by the subtraction from the scattering due to these samples of that due to sample B. The scatterers in sample B are possibly voids or any other structural inhomogenities detectable by SAXS. These structures are assumed to remain unchanged when filler particles are present in the SMRL sample. The subtraction results and the scattering curves for samples $A$ and B plotted in $\log$ (intensity) versus $\log (Q)$, are shown in Figure 1. Figure 2 shows the plot of the data in Figure 1 in terms of $\log$ (intensity) versus $Q^{2}$. The scattering curves in Figure 1 will be analysed based on Porod's laws and the scaling procedure, while in Figure 2 based on Guinier's law. This will be discussed in the later part of the text.

A few selected TEM-micrographs (Figures 3 and 4) obtained using two different instruments described above are only shown as

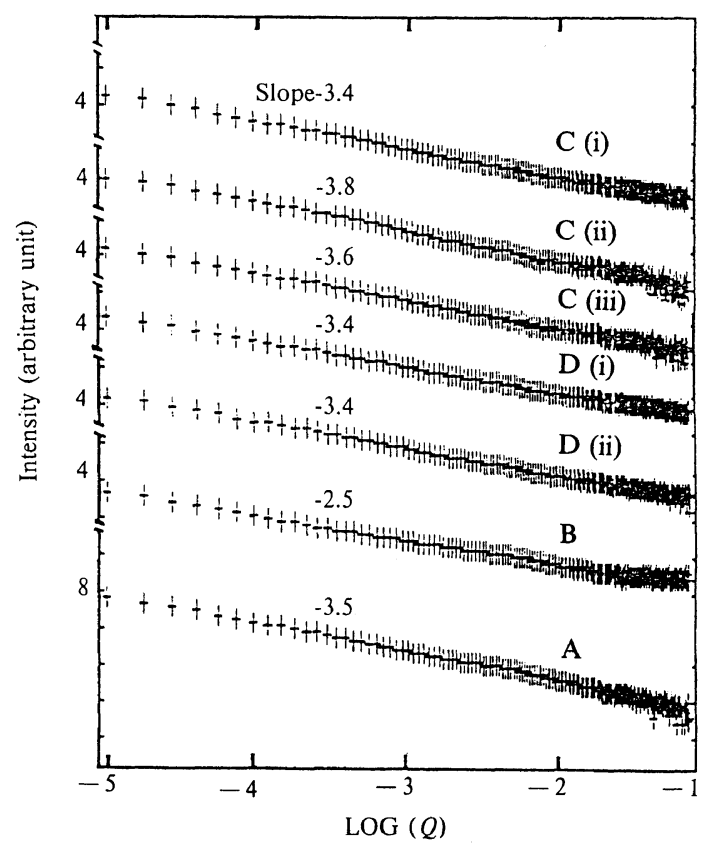

Figure 1. Double log plot of the scattering data. Label for samples are shown in Table I. all of them appear to be almost identical.

The Porod slopes from Figure 1 are listed in Table I. It is clear that the Porod formula shown in eq 1 obeyed by all of the data but with fractional values of $n$. All SMRL samples containing carbon black filler appear to have the scattering data which give the $n$ values ranging from 3.4 to 3.8 . The $n$ value for scattering due to the isolated carbon black falls in between these values. The small difference in these $n$ values may be the same order as the experimental error. These values which are close to each other, may indicate that, in the size range measured there is no change in the shape of the aggregates of carbon black particles before, and after incorporation in natural rubber with different treatment procedures shown in Table I.

The scaling procedure applied to scattering curves in Figure 1, except for SMRL sample without filler particles, gives a satisfactory

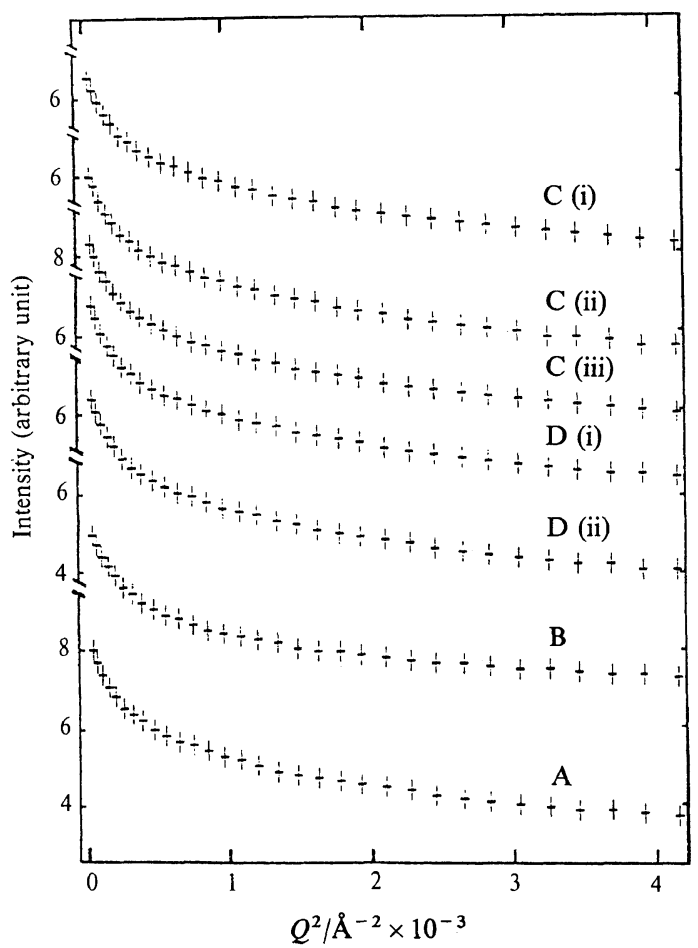

Figure 2. Guinier plot of the scattering data. Label for samples are shown in Table I. 


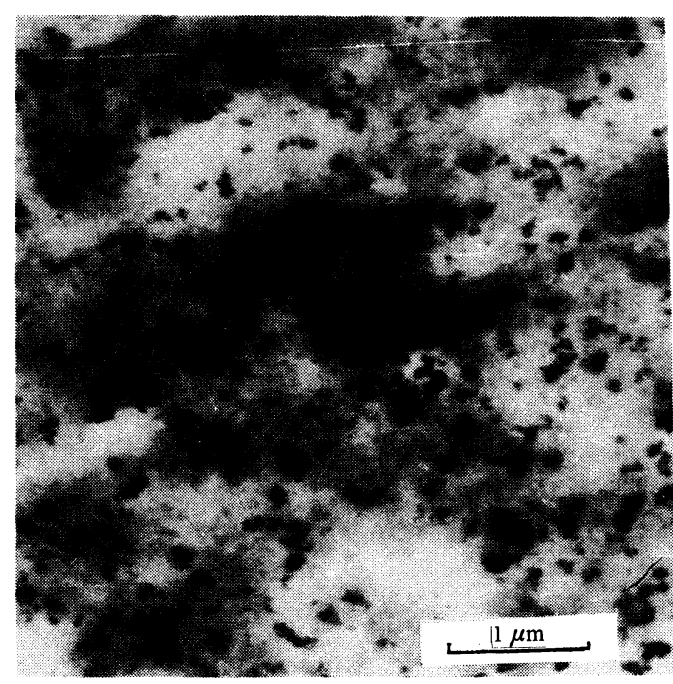

Figure 3. Transmission electron micrograph for sample C(iii). Low micrograph used to estimates the aggregate number per sq. $\mu \mathrm{m}$, prepared by Rubber Research Institute of Malaysia.

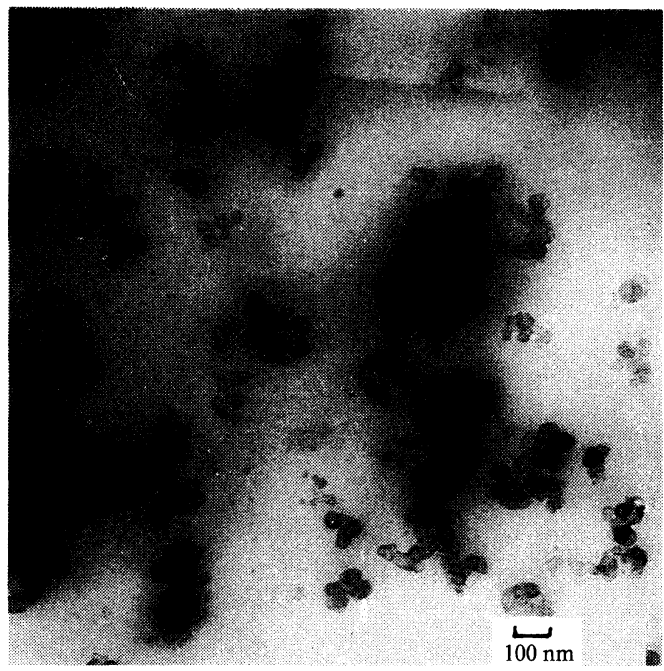

Figure 4. Transmission electron micrograph for sample C(iii). High magnification micrograph prepared by University of Sydney.

superposition. This, which was achieved through ordinate shifts only, indicates that the average sizes of carbon black aggregates remain unchanged after incorporation in natural rubber with different treatment procedures shown in Table I.
All of the TEM micrographs seem to show that aggregates of cabon black consists of dense parts located not necessarily at the middle of the aggregate, and 'arms' connected to these dense parts. The number of these dense parts in each aggregate varies, depending on the size of the aggregate. The dense parts appear to have a spherical shape whereas the arms structure behave like a fractal. TEM micrographs also shown that the aggregates have a broad size distribution with sizes approximately ranging from $10 \mathrm{~nm}$ to $400 \mathrm{~nm}$ for all samples investigated.

As in the TEM analysis, SAXS data also suggest the broad size distribution for carbon black aggregates. This is shown by the Guinier plots (Figure 2), which gives a curved slope of scattering curves instead of a straight line, representing the aggregates are monodisperse.

The structure of arms discussed above may be responsible for the fractional values of $n$, and without these arms, $n$ values obtained would probably become 4 , representing the spherical shape of the dense part of the aggregate. If this is true, we expect that the absence of these dense part would give $n$ values between -1 to -3 , which is the case for a full fractal behavior exhibited by those arms, see Theoretical section. In this context, it is therefore reasonable to assume that the observed $n$ values between 3.4 to 3.8 eventually arise from the contributions of both spherical-shape and fractal like structures that belong to the aggregates of carbon black particles before and after incorporation into rubber.

Acknowledgments. One of the authors Dr. Mohamad bin Deraman, wishes to thank Dr. C. Howard for his cooperation during his attachment with Neutron Diffraction Group at Australian Nuclear Science and Technology Organization (ANSTO), Australia. I also wish to thank Australia International Development Assistance Bureau (AIDAB) for financial assistance during my stay in Australia. We acknowledge Mr. Lorraine van der Lubbe of 
University Sydney who prepared the samples for electron microscopic measurements, and Dr. Tim White for electron microscopic measurements. We also acknowledge $\mathrm{Mr}$. Mohd bin Harun for his discussion and suggestion in this work.

\section{REFERENCES}

1. A. I. Medalia, in "Carbon Black-Polymer Composite," E. K. Sichel ed., Marcel Dekker, New York, 1982.

2. A. I. Medalia and F. A. Heckman, Carbon, 7, 567
(1969).

3. R. J. Young, D. H. Al-Khudhairy, and A. G. Thomas, J. Mater. Sci., 21, 1211 (1986).

4. P. W. Schmit, C. Y. Kwak, and M. Kalliat, J. Am. Chem. Soc., 28, 75 (1984).

5. A. Guinier and G. Fournet, "Small-Angle Scattering of X-Rays,” Wiley, New York, 1955.

6. G. Porod, Kolloid Z., 124, 93 (1951).

7. J. E. Martin, J. Appl. Cryst., 19, 25 (1986).

8. P. Fratzl, J. L. Lebowitz, J. Marrd, and M. H. Kalos, Acta Metal., 31, 1849 (1983).

9. M. Deraman, W. Hein, W. Wagner, and D. Juul Jensen, J. Sci. Nuclear, Malaysia, 5(2), 33 (1987). 